\title{
High-Linearity High-Resolution Time-of-Flight Linear-Array Digital Image Sensor Using Time-Domain Feedback
}

\author{
Juyeong Kim ${ }^{1} ®$, Keita Yasutomi ${ }^{2}$, Keiichiro Kagawa ${ }^{1,2}$ and Shoji Kawahito ${ }^{1,2, *}$ \\ 1 Graduate School of Science and Technology, Shizuoka University, Hamamatsu, Shizuoka 432-8011, Japan; \\ jkim@idl.rie.shizuoka.ac.jp (J.K.); kagawa@idl.rie.shizuoka.ac.jp (K.K.) \\ 2 Research Institute of Electronics, Shizuoka University, Hamamatsu, Shizuoka 432-8011, Japan; \\ kyasu@idl.rie.shizuoka.ac.jp \\ * Correspondence: kawahito@idl.rie.shizuoka.ac.jp; Tel.: +81-53-478-1313
}

Citation: Kim, J.; Yasutomi, K.;

Kagawa, K.; Kawahito, S.

High-Linearity High-Resolution Time-of-Flight Linear-Array Digital Image Sensor Using Time-Domain Feedback. Sensors 2021, 21, 454 https://doi.org/10.3390/s21020454

Received: 28 October 2020 Accepted: 6 January 2021 Published: 11 January 2021

Publisher's Note: MDPI stays neutral with regard to jurisdictional clai$\mathrm{ms}$ in published maps and institutional affiliations.

Copyright: $\odot 2021$ by the authors. Licensee MDPI, Basel, Switzerland. This article is an open access article distributed under the terms and conditions of the Creative Commons Attribution (CC BY) license (https:// creativecommons.org/licenses/by/ $4.0 /)$.

\begin{abstract}
This paper presents a high-linearity high-resolution time-of-flight (ToF) linear-array digital image sensor using a time-domain negative feedback technique. A coarse ToF measurement loop uses a 5-bit digital-to-time converter (DTC) and a delayed gating-pulse generator for time-domain feedback to find the zero of the difference between ToF and the digital estimate of the gating-pulse delay while maintaining a constant operating point of the analog readout circuits. A fine ToF measurement uses a delta-sigma modulation (DSM) loop using the time-domain feedback with a bit-stream signal form. Because of the self-contained property of the DSM for low distortion and noise exploited by the oversampling signal processing, the proposed technique provides high-linearity and high-range resolution in the fine ToF measurement. A prototype ToF sensor of $16.8 \times 16.8 \mu \mathrm{m}^{2}$ two-tap pixels and fabricated in a $0.11 \mu \mathrm{m}(1 \mathrm{P} 4 \mathrm{M})$ CMOS image sensors (CIS) process achieves $+0.9 \% /-0.47 \%$ maximum nonlinearity error and a resolution of $0.24 \mathrm{~mm}$ (median) for the measurement range of 0-1.05 m. The ToF sensor produces an 11-bit fully digital output with a ToF measurement time of $22.4 \mathrm{~ms}$.
\end{abstract}

Keywords: CMOS image sensor (CIS); short-pulse; time-of-flight (ToF); indirect ToF; depth sensing

\section{Introduction}

Among the various functions of CMOS image sensors (CISs), time-of-flight (ToF) range image sensors are receiving much attention for new markets and applications of CISs, including consumer, industrial, and scientific applications [1-3]. ToF range sensor applications require high precision, accuracy, linearity, and tolerance to ambient light. Direct-type ToF imagers using a single-photon avalanche diode, and all-digital-domain processing are excellent for high-accuracy ToF [4-7]. However, it requires complex hardware, specifically, if there is a need for extremely high-resolution and tolerance to ambient light. Indirect-type ToF imagers have an advantage of small pixel size, less circuit complexity, and relatively reliable range resolution, specifically for range measurements of a few meters. Two types of indirect ToF imaging methods exist, depending on the waveform of the modulated light. One is continuous wave (CW)-based [8-15], and the other is short pulse (SP)-based [16-24]. The SP-based indirect ToF has a better tolerance to ambient light because the light power is concentrated on the short pulse, and the charge-draining function of the ToF pixel reduces the influence of the ambient light. However, because of the analog-domain processing for ToF measurement, the SP-based indirect ToF sensor suffers from various analog imperfections, such as the nonlinearity of the pixel source-follower amplifier, the distortion of the waveform of the light pulse, finite photo-carrier response time in the photodiode, and the distortion of the gating pulse for demodulation. The full-well capacity of the pixel limits the range resolution or depth noise of indirect ToF sensors if the photon-shot noise is the liming factor of the range resolution [3]. Using a short pulse is effective for improving the range resolution. High-resolution ToF sensors designed with a pixel using a very 
short light pulse lower than $100 \mathrm{ps}$ and a large full-well capacity larger than $1 \mathrm{M}$ electrons have range resolutions of sub-millimeter [25] and sub-100 micrometer [26]. Because of the short pulse, the nonlinearity and skew of the gating pulses become significant issues to be solved. Complicated off-line processing for nonlinearity correction and the on-chip skew calibration circuits are necessary for implementing a linear array ToF sensor.

To address to the issues of the SP-based indirect ToF sensors, this paper proposes an SP-based indirect ToF image sensor using the time-domain negative feedback technique. To the best of our knowledge, this work is the first attempt at a indirect ToF image sensor using a time-domain negative feedback technique. In the conventional indirect ToF image sensors, open-loop analog interface circuits and a successive analog-to-digital converter (ADC) are used. Techniques for improving their linearity and range resolution, respectively, are based on the design effort of the open-loop analog readout circuits like source followers and having a high demodulation frequency (or short pulse width) and high-full-well capacity $[10,26]$. A negative feedback technique in analog circuits and systems is known as an effective way for improving the nonlinearity, frequency response, and stability [27,28]. In the proposed design, the negative feedback technique is used at the time-domain for coarse and fine ToF measurements. The negative feedback used for coarse ToF measurements is based on finding zero of the ToF difference from the delay of gating pulses generated by a digital-to-time converter (DTC), effectively improving the linearity by maintaining a constant operating point of analog readout circuits. In fine ToF measurements, a first-order delta-sigma modulation (DSM) loop using time-domain feedback is used. The DSM loop using one-bit analog-to-digital converter (ADC) and one-bit digital-to-analog converter, which is popular for audio ADCs, has a self-contained property of low distortion and noise using oversampling signal processing [29-31]. It provides high-linearity and range resolution in ToF measurements if it is applied as the DSM using the time-domain feedback. A prototype chip for the concept proof is implemented, and the linearity and resolution are characterized.

The rest of the paper is organized into Section 2 describing the principle of ToF measurements using time-domain feedback. Section 3 describes the circuit design, and Section 4 provides the results of the ToF measurement system implementation and measurements. Section 5 presents the concluding remarks.

\section{ToF Measurement Using Time-Domain Feedback}

Figure 1 shows a system diagram of the proposed sensor. The light pulse emitted from the light source is reflected onto the object and converted into an electrical signal by a photodiode (PD) and a demodulator. The time-domain negative feedback loop consists of a demodulator, analog processing unit (APU), 1-bit ADC, digital processing unit (DPU), and digital-to-time converter (DTC). This system measures the ToF with high linearity and resolution. To cover the wide range of ToF measurements while maintaining high-linearity and range resolution, the system in Figure 1 works with a coarse and fine measurement step. To realize the coarse and fine measurements with the time-domain negative feedback loop, each APU and DPU have two switchable functions. In the coarse measurement, a buffer amplifier and digital counter are used for the APU and DPU, respectively, working as an incremental ToF-to-digital conversion. In the fine measurement, an integrator and digital adder are used for the APU and DPU, respectively, working as a first-order DSM.

\subsection{Incremental Time-to-Digital Conversion for Coarse ToF Measurements}

Figure 2 shows an equivalent block diagram for coarse ToF measurements, consisting of a demodulator, buffer gain stage, 1-bit ADC, digital counter, and DTC. This system works as an incremental time-to-digital conversion (TDC). The demodulator senses the time position of ToF, and the demodulated charges are converted into a voltage signal at the buffer stage. With the time-domain feedback with DTC output, the voltage signal output of the demodulator with the buffer gain stage is expressed as 


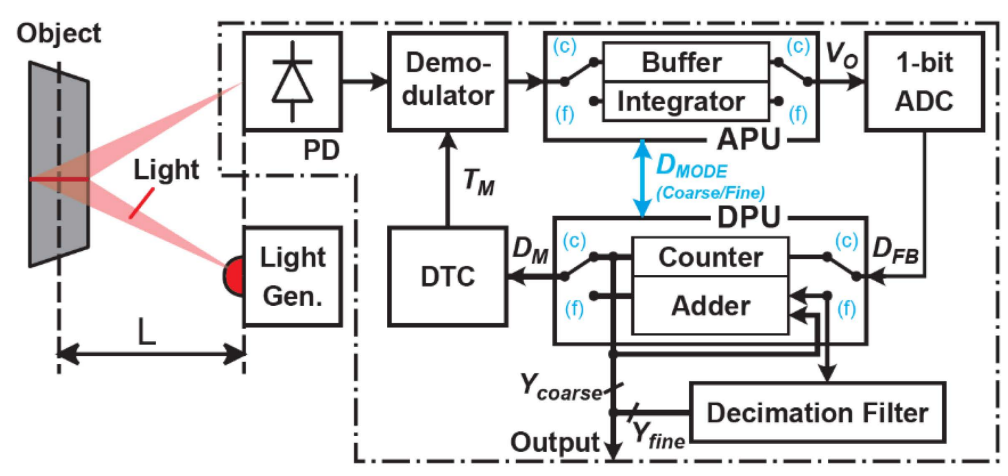

Figure 1. A system diagram of the proposed sensor.

$$
V_{O}=K_{B U F} K_{M}\left(T o F-T_{M}\right),
$$

where $K_{M}$ is the time-to-voltage conversion gain of the demodulator, $K_{B U F}$ is the voltage gain of the buffer, and $T_{M}$ is the DTC output in the time-domain. The 1-bit ADC quantizes the buffer output with a threshold of zero, and the increment code for the digital domain feedback, $D_{F B}$, is generated as

$$
D_{F B}=\left\{\begin{array}{ll}
0 & \left(V_{O}<0\right) \\
1 & \left(V_{O} \geq 0\right)
\end{array}\right. \text {. }
$$

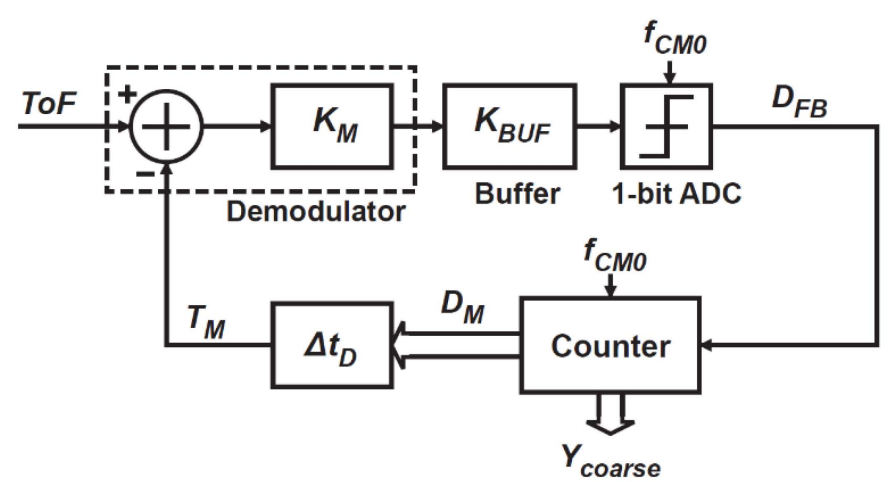

Figure 2. An equivalent block diagram for coarse time-of-flight (ToF) measurements.

The $D_{F B}$ is given to a counter where the number of 1 's is counted, and the counter output at the $n$-th step, $D_{M}(n)$, is expressed as

$$
D_{M}(n)=D_{M}(n-1)+D_{F B}
$$

The delay of DTC output at the $n$-th step, $T_{M}(n)$, which is the time-domain feedback to the demodulator, is given by

$$
T_{M}(n)=\Delta t_{D} \cdot D_{M}(n),
$$

where $\Delta t_{D}$ is the conversion factor of DTC, which is equal to the DTC unit delay step. Figure 3 shows a simplified conceptual operating waveform and the ToF quantization error. To coarsely measure ToF using the incremental TDC, the initial value of the counter output, $D_{M}(0)$, is set to zero so that $T_{M}$ is initially set to zero and $T_{M}$ is gradually approximated step-by-step to ToF, expressed as a set of the above equations. While $D_{F B}$ is 1 , the DTC delay is incremented. When $T_{M}$ becomes larger than ToF, $D_{F B}$ becomes 0 and the DTC 
output delay increment is stopped. When this happens at the $n_{C}$-th step, the counter output is expressed as

$$
D_{M}\left(n_{C}\right)=n_{C}
$$

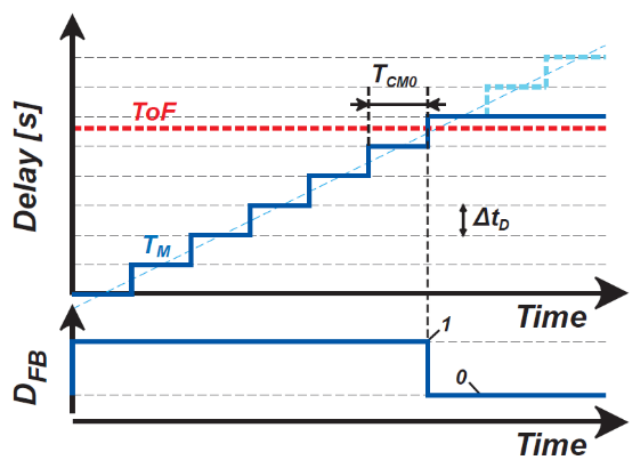

(a)

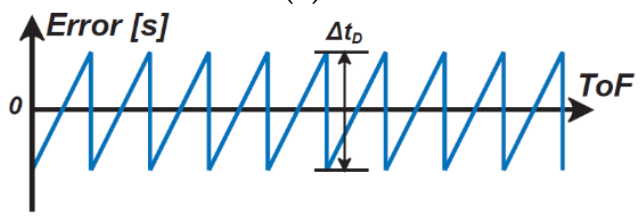

(b)

Figure 3. (a) Simplified conceptual operating waveform and (b) the quantization error of the ToF for coarse measurements.

The counter output at the final step, $D_{M}\left(N_{C}\right)$, is also $n_{C}$ because the counter output is not incremented after the $n_{C}$-th step, where $N_{C}$ is the maximum number of DTC delay steps. The DTC output at the final step, $T_{M}\left(N_{C}\right)$, is given by $\Delta t_{D} \cdot n_{C}$. The difference between the ToF and $T_{M}\left(N_{C}\right)$ is the error of the measured ToF because of the incremental TDC in the coarse ToF measurement mode. The error is maximal within the range of $-\Delta t_{D} / 2$ to $\Delta t_{D} / 2$ (Figure $3 \mathrm{~b}$ ). The maximum ToF measurement range is $T_{M, M a x}$, given by

$$
T_{M, \operatorname{Max}}=N_{C} \cdot \Delta t_{D}
$$

The output of the coarse ToF measurements, $Y_{\text {coarse, }}$ is the final code stored in the counter as

$$
Y_{\text {coarse }}=n_{C} \text {. }
$$

The time required for doing the coarse ToF measurement time, $T_{C M}$, is expressed as $T_{C M}=N_{C} \cdot T_{C M 0}$ if the time required for doing one step for the gradual approximation is $T_{C M 0}$.

\subsection{DSM for Fine ToF Measurements}

Figure 4 shows an equivalent block diagram for fine ToF measurements, employing a first-order delta-sigma modulation (DSM) with time-domain negative feedback. Though the use of higher-order DSMs has more efficient improvement of SNR to the oversampling ratio used, the first-order DSM is employed for an area-efficient column-wise implementation with a limited column pitch. In this fine ToF measurement mode, an integrator and adder are used for the APU and DPU, respectively. The adder adds coarse ToF measurement results stored in the counter, and the DSM finely measures the ToF difference from a coarse estimate of it, $\triangle T O F$. From Figure 4 , the DSM output $D_{F B}[z]$ is expressed as

$$
D_{F B}[z]=E_{q}(z)+\frac{K_{I N T} K_{M}}{1-z^{-1}} D(z),
$$


where $K_{I N T}$ is the gain of the integrator, $E_{q}$ is the quantization noise of the 1-bit ADC, and $D(z)$ is the difference of $\triangle T O F$ to the time domain feedback, expressed as

$$
D(z)=\Delta \operatorname{TOF}(z)-\Delta t_{D} z^{-1} Y(z) .
$$

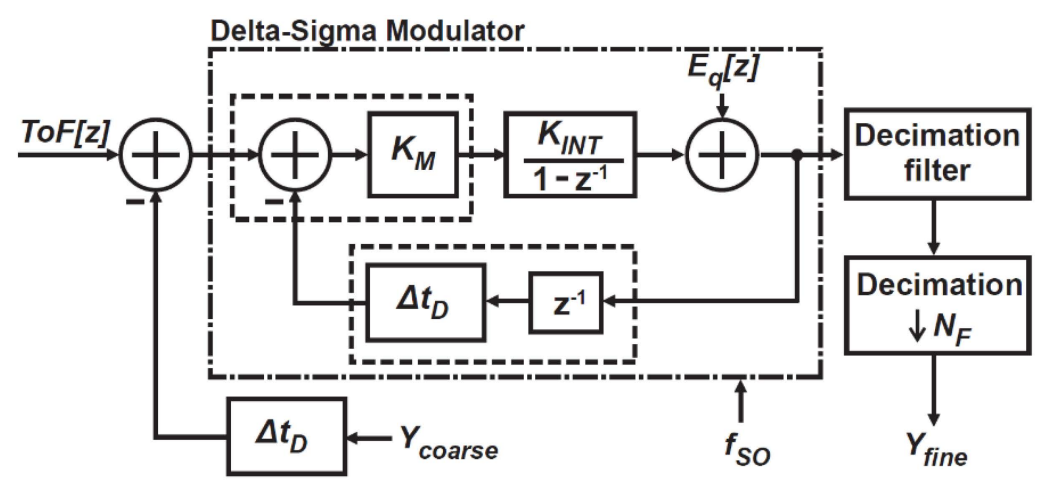

Figure 4. An equivalent block diagram for fine ToF measurements.

By substituting Equation (9) into (8), the overall system response in the z-domain for the DSM part of Figure 4 is expressed as

$$
D_{F B}[z]=\frac{K_{I N T} K_{M}}{1-\left(1-K_{I N T} K_{M} \Delta t_{D}\right) z^{-1}} \Delta T o F[z]+\frac{1-z^{-1}}{1-\left(1-K_{I N T} K_{M} \Delta t_{D}\right) z^{-1}} E_{q}[z],
$$

where $K_{I N T}$ is the gain of the integrator, and $E_{q}$ is the quantization noise of the 1-bit ADC. The conventional first-order DSM using a 1-bit ADC shows that the loop gain of the modulator is unity if the modulator is stably operated [31]. Therefore, the condition $K_{I N T} K_{M} \Delta t_{D}=1$ is met, and the equation is simplified as

$$
D_{F B}[z]=\frac{1}{\Delta t_{D}} \Delta T o F[z]+\left(1-z^{-1}\right) E_{q}[z] .
$$

From Equation (11), transfer functions to signal and quantization noise are obtained. The signal transfer function, the relationship between $D_{F B}[z]$ and $\triangle T O F[z]$, is the same as the inverse of the DTC delay step. The noise transfer function (NTF), the relationship between $D_{F B}[z]$ and $E_{q}[z]$, is $1-z^{-1}$. In order to estimate the quantization noise using the DSM, it is useful to find the squared magnitude of NTF in the frequency domain [30] by setting $z=e^{j 2 \pi f T_{S O}}$, given by

$$
\left|N T F\left(e^{j 2 \pi f T_{S O}}\right)\right|^{2}=\left[2 \sin \left(\pi f / f_{S O}\right)\right]^{2},
$$

where $f$ is the frequency, $T_{S O}$ is the period of the oversampling, and $f_{S O}\left(=1 / T_{S O}\right)$ is the oversampling frequency.

Figure 5 shows the power spectrum density (PSD) due to the shaped quantization noise through the NTF given by Equation (10). The shaped quantization noise is reduced using a digital low-pass filter (LPF) with a cutoff frequency of $f_{L P F}$. Then, the ratio of the square root of the residual noise power (the red colored area) to the entire quantization noise (the sum of the red and blue colored areas) gives the increase of the effective number of bits (ENOB) in the fine TDC. With the oversampling ratio denoted by $N_{F}$ and given by $f_{S O} / 2 f_{L P F}$, the area ratio of red-colored to (red + blue)-colored is approximately $1 / N_{F}^{3}[30,31]$. Then the ENOB increase using an ideal LPF denoted by $\triangle E N O B_{i}$ is given by

$$
\triangle E N O B_{i}=\log _{2} N_{F}^{3 / 2}[\mathrm{bit}]=1.5 \log _{2} N_{F}[\mathrm{bit}] .
$$




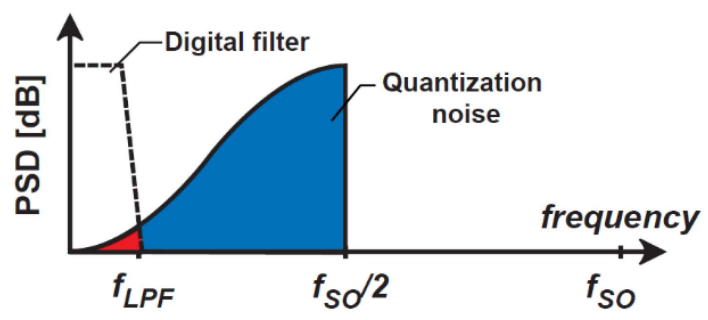

Figure 5. Noise transfer function of delta-sigma modulation.

In this proposed technique using the first-order DSM, $\triangle E N O B_{i}=3$ [bit] is obtained for $N_{F}$ of 4 . However, this is the case that an ideal low-pass filter, which has very steep cutoff, is used. In the actual implementation, a very simple counter that counts the number of 1's in the bit-stream is used for the low-pass filtering. Because the counter does not have steep cutoff and its frequency response is like a moving average filter, the residual quantization noise power after low-pass filtering is $1 / N_{F}{ }^{2}$ of the entire quantization noise. The increase in the effective number of bits (denoted by $\triangle E N O B_{1}$ ) in this case is given by

$$
\triangle E N O B_{1}=\log _{2} N_{F}[\mathrm{bit}] .
$$

In the actual implementation, $N_{F}=64$ (or 66 for error reduction) and a counterbased low-pass filter is used, and the ENOB increase in the fine TDC implementation is 6 bits. From Equation (14), doubling the oversampling ratio $N_{F}$ can increase the ENOB by another one bit, while having the penalty of doubling the processing time of the fine ToF measurement. The filtered output of the DSM linearly responds to the $\triangle T O F$, and a fine TDC for fine ToF measurement is realized.

\subsection{Coarse-to-Fine ToF Measurements}

Figure 6 shows the conceptual operating waveforms of the proposed ToF measurement technique, including the incremental time-to-digital conversion and delta-sigma modulation for coarse and fine ToF measurements. After the coarse conversion, the incremental TDC output produces a coarse estimate of $\mathrm{ToF}, \Delta t_{D} n_{C}$. In subsequent fine conversion using the DSM, DTC output produces a waveform of bit-stream and takes two states of $\Delta t_{D} n_{C}$ and $\Delta t_{D}\left(n_{C}-1\right)$. The DSM output is low-pass filtered and down-sampled for decimation, and the fine ToF measurement, or a fine TDC output, $Y_{\text {fine }}$ is produced.

Figure 6 shows the filter output behavior when a digital counter (a first-order integrator) is used for the decimation filter. The filter output at the end of fine conversion is expressed as the number of 1's of the DSM output, and if it is $n_{F}$ and the total sampling number is $N_{F}$, the output of fine ToF measurements $Y_{\text {fine }}$ is expressed as

$$
Y_{\text {fine }}=n_{F} / N_{F} \text {. }
$$

$Y_{\text {fine }}$ takes the value from $0-1$. The output using coarse-to-fine cascaded ToF measurements $Y_{T O F}$ is given by

$$
Y_{\text {TOF }}=Y_{\text {coarse }}+Y_{\text {fine }}-1
$$

or

$$
Y_{T O F}=n_{C}+n_{F} / N_{F}-1
$$

If $N_{C}$ and $N_{F}$ are chosen as $2^{m_{C}}$ and $2^{m_{F}}$, this coarse-to-fine ToF measurement technique converts the ToF to a $\left(m_{C}+m_{F}\right)$-bit digital code as a result of incremental and DSM-based TDC techniques. 


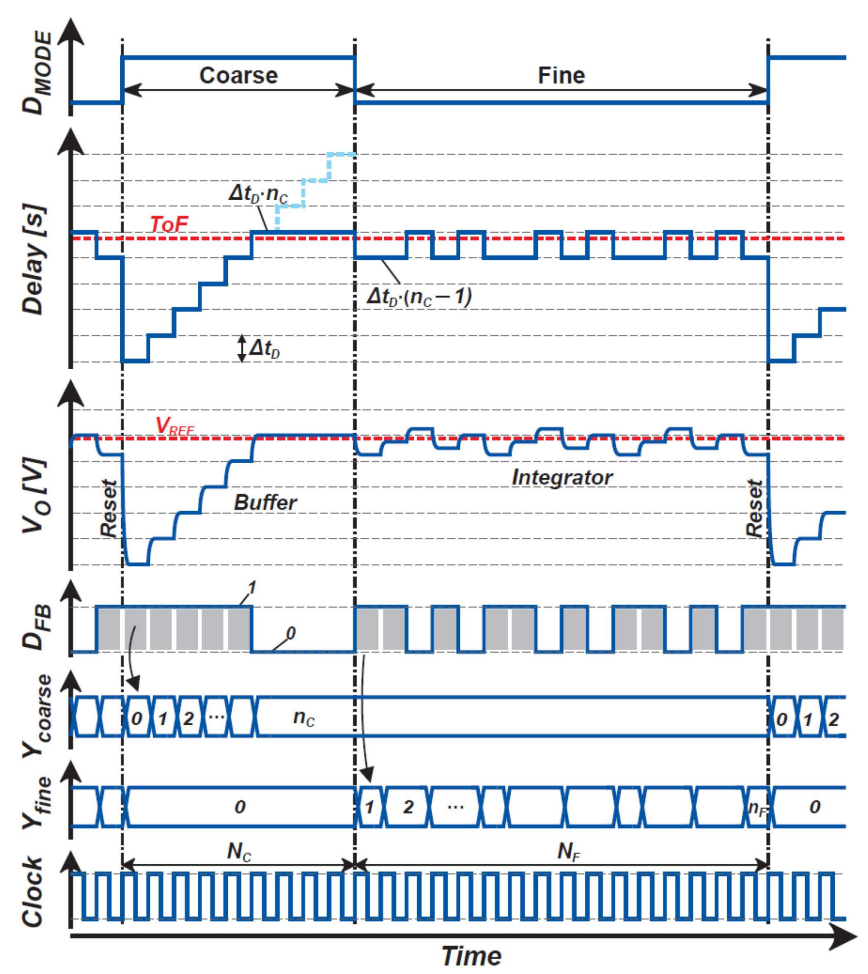

Figure 6. Conceptual operating waveform of the proposed system.

\section{Circuit Design}

\subsection{Photo-Signal Demodulator}

Figure 7 shows a circuit schematic of the photo-signal receiver and demodulator. Figure 8 shows the operation-timing diagram for the photo-signal receiver and demodulator. A PD in a pixel receives a periodic short-light pulse train and the generated electrons are transferred to a floating diffusion node $\left(\mathrm{FD}_{1}\right)$, another floating diffusion node $\left(\mathrm{FD}_{2}\right)$, or a drain, depending on the light pulse delay. The $\mathrm{FD}_{1}$ and $\mathrm{FD}_{2}$ are connected to the input of a fully differential charge-sensitive amplifier (CSA) to convert the modulated photo-charge to a differential voltage. The amplifier is a folded-cascode type with switched-capacitor common mode feedback (CMFB), having $77 \mathrm{~dB}$ gain and $53 \mathrm{MHz}$ unit gain bandwidth at a phase margin of 76 degrees. The charge transfer control gates $G_{1}, G_{2}$, and $G_{D}$ demodulate the generated photo-signal. When photons move to the PD while $G_{1}$ is set to a high voltage level, the generated photoelectron is transferred to $\mathrm{FD}_{1}$. The photoelectron generated when the $G_{2}$ is set to a high level is transferred to $\mathrm{FD}_{2}$, and when the $G_{D}$ is set to a high level, they are drained by connecting the drain terminal to a voltage source $V_{D}$. For ToF measurements with high-resolution, the switching time for this demodulation must be subnanoseconds. The photoreceiver and demodulator are designed to perform this function, and the sub-nanosecond carrier response is based on the design reported in [21] where the photoreceiver is implemented with a lateral drift PD. The $G_{1}$ and $G_{2}$ are implemented with lateral electric field modulation (LEFM) gates, and the $G_{D}$ is implemented with a MOS transfer gate [22]. The charges $Q_{1}$ and $Q_{2}$ are transferred to FD1 and FD2, respectively, because of the operation of LEFM gates. Because of the operation of the CSA, the charges $Q_{1}$ and $Q_{2}$ are mainly transferred and accumulated in two feedback capacitors $C_{S}$ in the CSA. Using a common-mode feedback (CMFB) technique to control the average level of the fully differential output to be equal to a common level, $V_{C O M}$, the output of the CSA, $V_{P 1}$, and $V_{P 2}$, is expressed as

$$
V_{P 1} \cong \frac{Q_{1}-Q_{2}}{2 C_{S}}+V_{C O M} \text { and }
$$




$$
V_{P 2} \cong \frac{Q_{2}-Q_{1}}{2 C_{S}}+V_{C O M}
$$

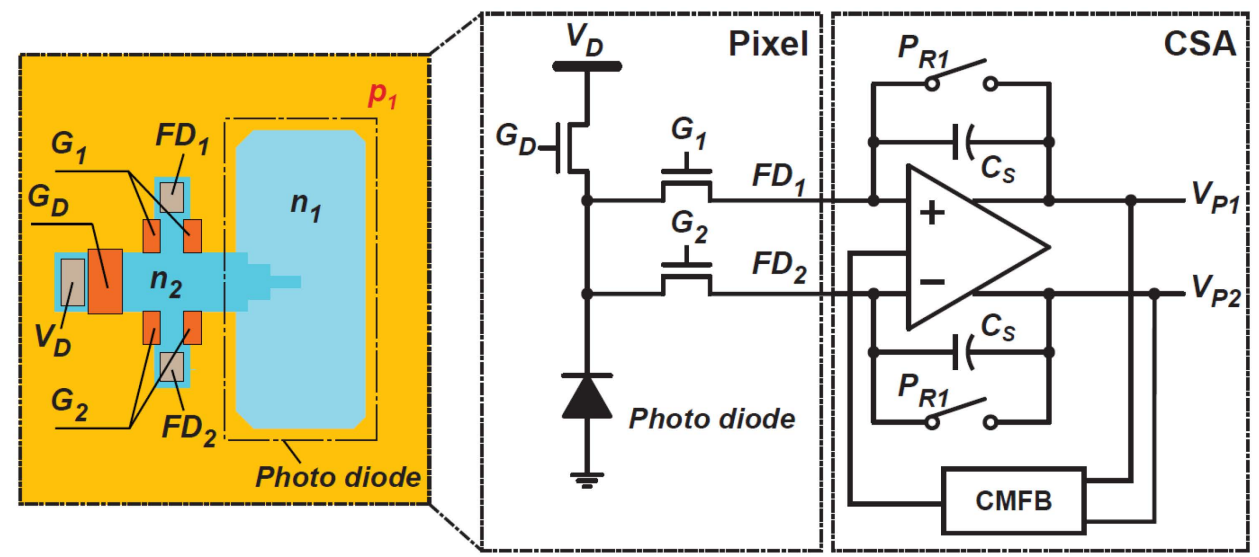

Figure 7. A circuit schematic of the photo-signal receiver and demodulator.

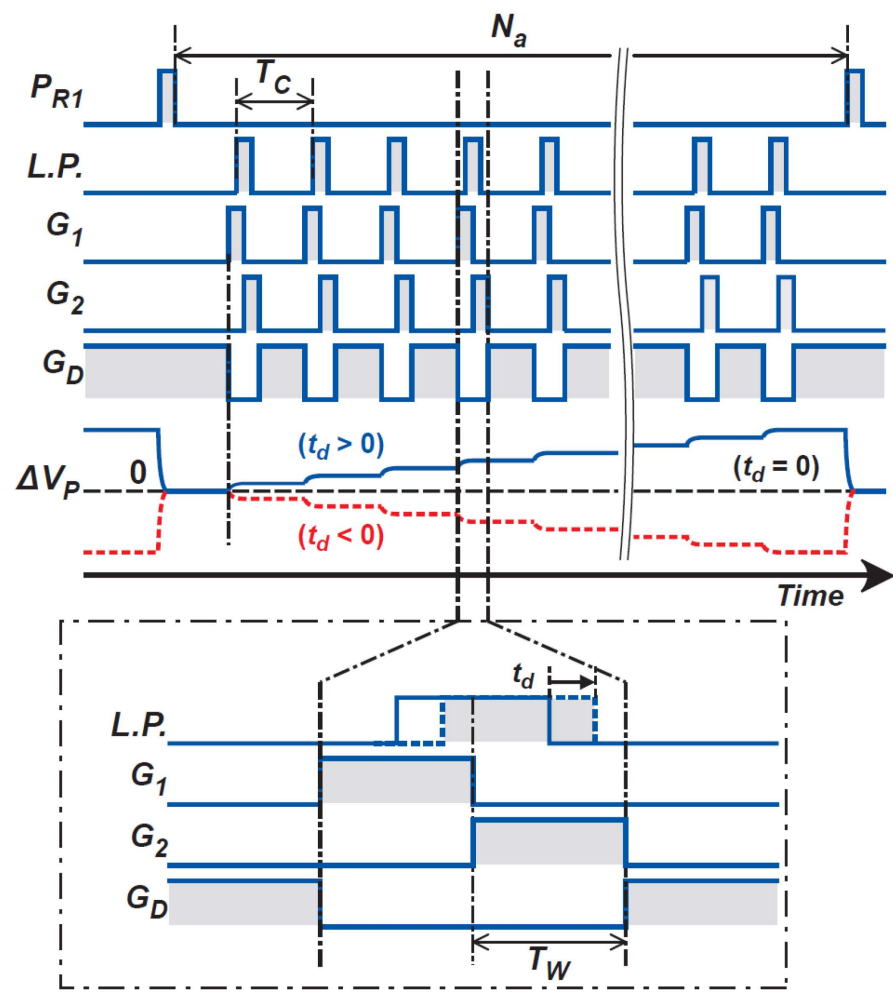

Figure 8. An operation-timing diagram for the photo-signal receiver and demodulator.

If the photo-generated charge occurs because of the signal light pulse for ToF measurements and ambient light, the $Q_{1}$ and $Q_{2}$ are expressed as

$$
\begin{gathered}
Q_{1} \cong Q_{S 1}+Q_{B} \text { and } \\
Q_{2} \cong Q_{S 2}+Q_{B}
\end{gathered}
$$

where $Q_{S 1}, Q_{S 2}$, and $Q_{B}$ are signal components of $Q_{1}$ and $Q_{2}$, respectively, and ambient light components. By using a fully differential amplifier with the $C M F B$, the differential output $\Delta V_{P}=V_{P 1}-V_{P 2}$ is given by

$$
\Delta V_{P}=V_{P 1}-V_{P 2} \cong \frac{Q_{S 1}-Q_{S 2}}{C_{S}} .
$$


The differential CSA output cancels the ambient light components and is proportional to the difference in the signal charge $Q_{S 1}-Q_{S 2}$. The charge-to-voltage conversion gain $G_{C}$ is given by

$$
G_{C} \cong \frac{\Delta V_{P}}{Q_{S 1}-Q_{S 2}}=\frac{1}{C_{S}} \text {. }
$$

Because a small number of photons are included in one short light pulse, the signal is intensified by receiving the light pulse periodically with many cycles. In Figure 8 , the behavior of $\Delta V_{P}$ is shown for three cases of the relative delay of light pulse $t_{d}$. If $t_{d}$ equals 0 and the photoelectrons are equally shared in $Q_{S 1}-Q_{S 2}, \Delta V_{P}$ takes 0 . If $t_{d}>0$, the CSA output signal gradually increases with positive polarity. If $t_{d}<0$, the CSA output gradually increases but with negative polarity. Therefore, the demodulator with the CSA can be used for measuring relative light pulse delays because of ToF. The CSA output is sampled by the next stage after the CSA output is sufficiently intensified, and the charge in $C_{S}$ is reset by turning on switch $P_{R 1}$ to prepare for the next cycle.

\subsection{Analog Processing Unit for Fixed-Gain Amplifier and Integrator}

Figure 9 shows a circuit schematic of the APU for a fixed-gain amplifier and integrator that are used for coarse and fine ToF measurements, respectively. In the coarse ToF measurement, the APU performs as a buffer to carry a photo-signal to the comparator at every feedback cycle. It is implemented using a fully differential switched-capacitor (SC) amplifier where the ratio of capacitance used for the input and feedback path sets the amplifier gain. It works with two operation phases. In the first phase, input capacitors $C_{I}$ sample the differential input signal by turning on switches $P_{R 1 D}$. During that time, the charge in feedback capacitors $C_{F}$ is reset by turning on switches $P_{R 2}$. In the second phase, the switches $P_{R 1 D}$ and $P_{R 2}$ are turned off, and switches $P_{S}$ are turned on. The charges sampled in $C_{I}$ is transferred to $C_{F}$ so that the capacitance ratio amplifies the input signal. The gain of the buffer, i.e., the gain of the differential output to its differential input $A_{I}\left(=\Delta V_{O} / \Delta V_{P}\right)$, is expressed as

$$
A_{I}=\frac{\Delta V_{O}}{\Delta V_{P}}=\frac{C_{I}}{C_{F}}
$$

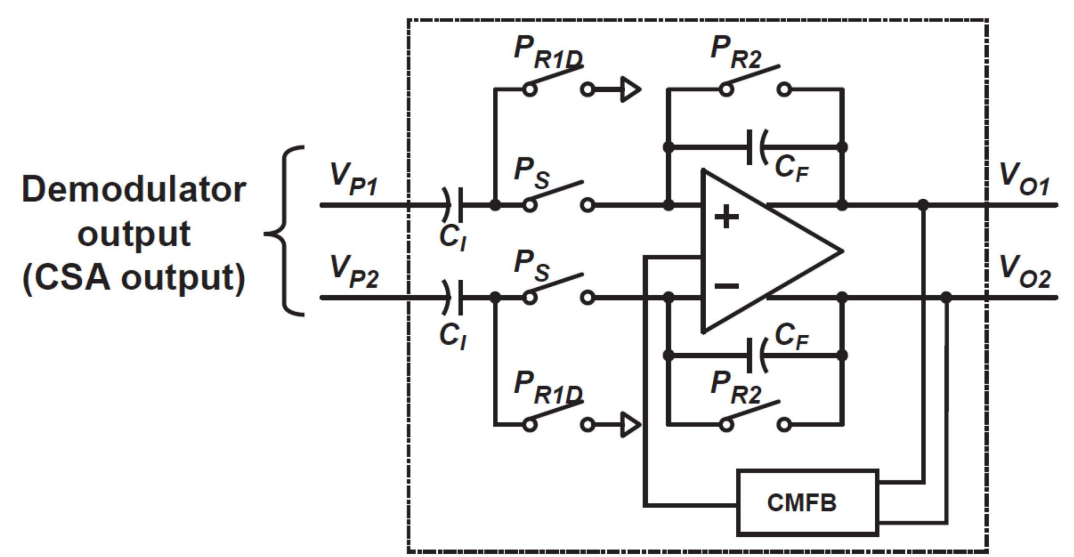

Figure 9. A circuit schematic of the analog processing unit (APU) for a fixed-gain amplifier and integrator.

In the fine ToF measurement, the APU performs as a fully differential SC integrator. In this operation mode, the switches $P_{R 2}$ are turned on for resetting the charge in $C_{F}$ at the beginning. Input signal sampling by $C_{I}$ and $P_{R 1 D}$ and charge transfer to $C_{F}$ by switches $P_{S}$ 
are repeated to conduct SC integration. The final output after repeated SC integration with $N_{F}$ cycles, $\Delta V_{O}\left(N_{F}\right)$, is given by

$$
\Delta V_{O}\left(N_{F}\right) \cong A_{I} \sum_{i=1}^{N_{F}} \Delta V_{P}(i),
$$

where $\Delta V_{P}(i)$ is the $i$-th differential input.

\subsection{DTC and Gating-Pulse Generator}

Figure 10 shows the DTC design and gating-pulse generator for ToF measurements with time-domain feedback. A digitally controlled delay line (DCDL) generates a delay time of an integer multiple of a unit delay, $\Delta t_{D}$, and is configured with 32 stages for a 5-bit resolution of DTC. Figure 11 shows the operation waveform of DTC and gatingpulse generator. The DTC output generates the three-phase gating pulses, $G_{1}, G_{2}$, and $G_{D}$, by connecting 3 DCDLs, each of which has a 5-bit counter, in series, and the three 5-bit DCDL outputs are compared with $D_{M}$, which is the DPU output in Figure 1 . The DCDL is triggered by a start pulse $T_{S}$. When $T_{S}$ is applied to the DCDL, three DCDLs start to generate a 5-bit binary counting code with the unit cycle time of $\Delta t_{D}$ (Figure 11). By comparing the three DCDL outputs with $D_{M}$, the gating-pulse generator generates a set of waveforms for $G_{1}, G_{2}$, and $G_{D}$, which is used for the photo-signal demodulator (Figure 7).

\section{Digital to Time Converter Gating-pulse generator}

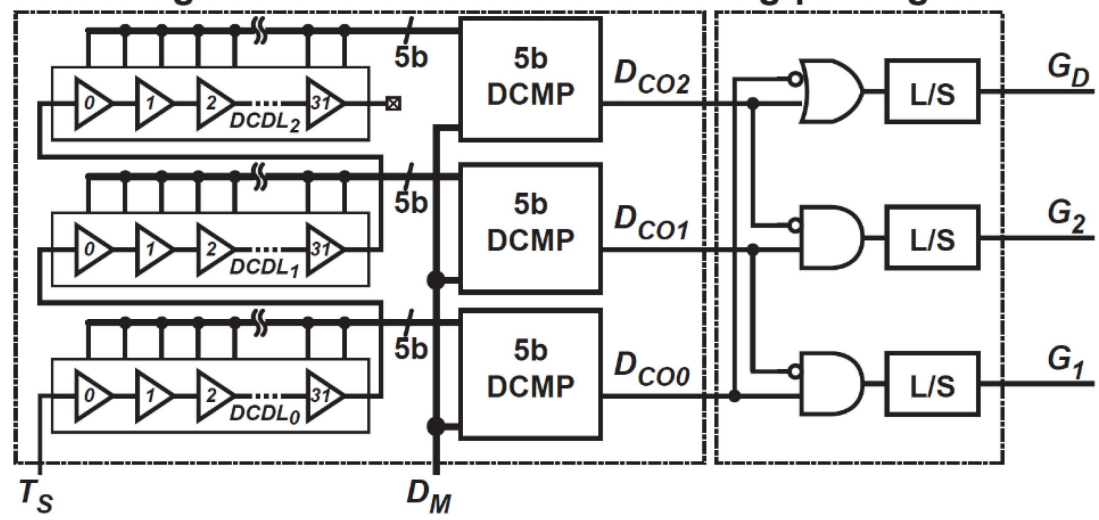

Figure 10. A circuit schematic of the digital-to-time converter and gating-pulse generator.

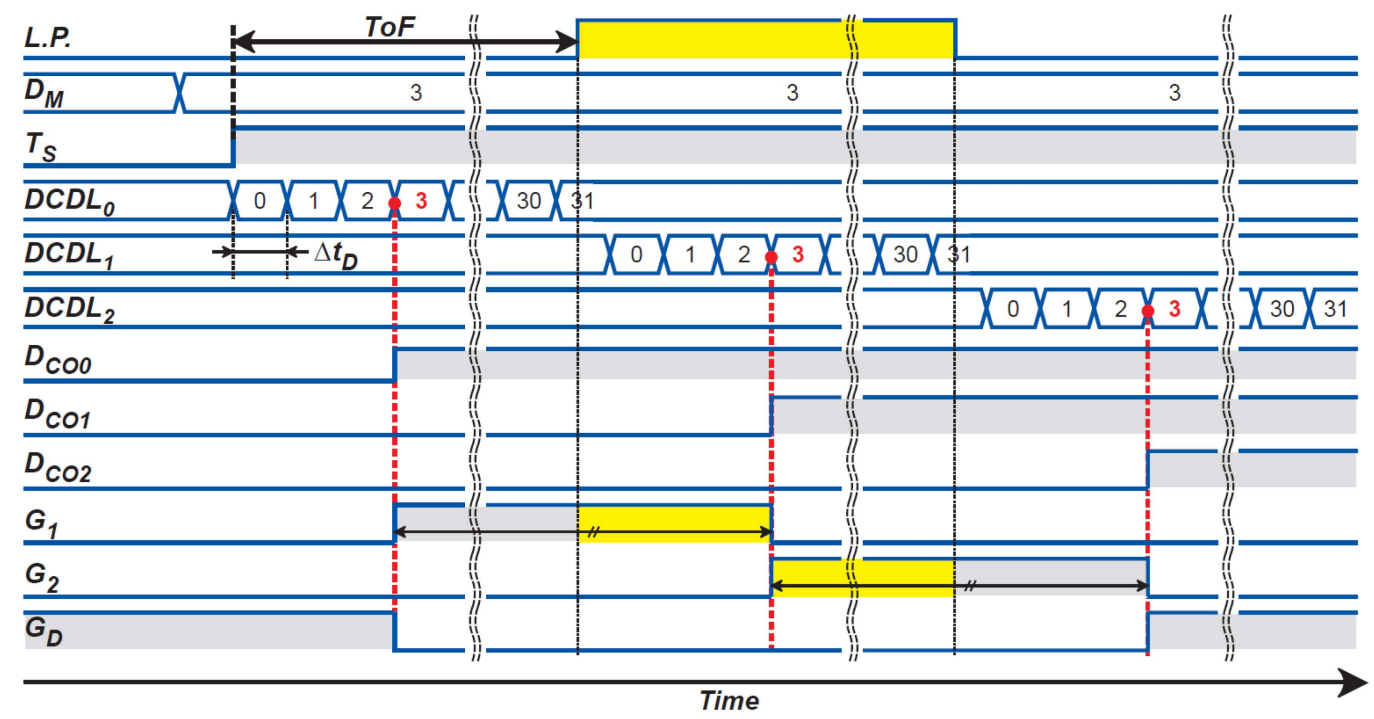

Figure 11. Conceptual operation waveform of the digital-to-time converter and gating-pulse generator. 


\section{Implementation and Measurement}

Figure 12 shows a photomicrograph of the prototype chip implemented in $0.11 \mu \mathrm{m}$ (1P4M) CIS technology. The chip includes $10 \times 3$ pixels with the demodulator, 10 units of a set consisting of a DCDL, a 5-bit digital comparator (DCMP), a demodulation gate driver, and an APU. The pixel size is $16.8 \times 16.8 \mu \mathrm{m}^{2}$, the pitch of the readout circuit channel is $16.8 \mu \mathrm{m}$, and the chip core size is approximately $0.232 \mathrm{~mm}^{2}$. A multiplexer chooses the APU output to evaluate the characteristics of one channel. External components of a fieldprogrammable gate array (FPGA), 1-bit comparator, light pulse generator, digital delay controller, and PC interface are used for implementing the proposed system in Figure 1.

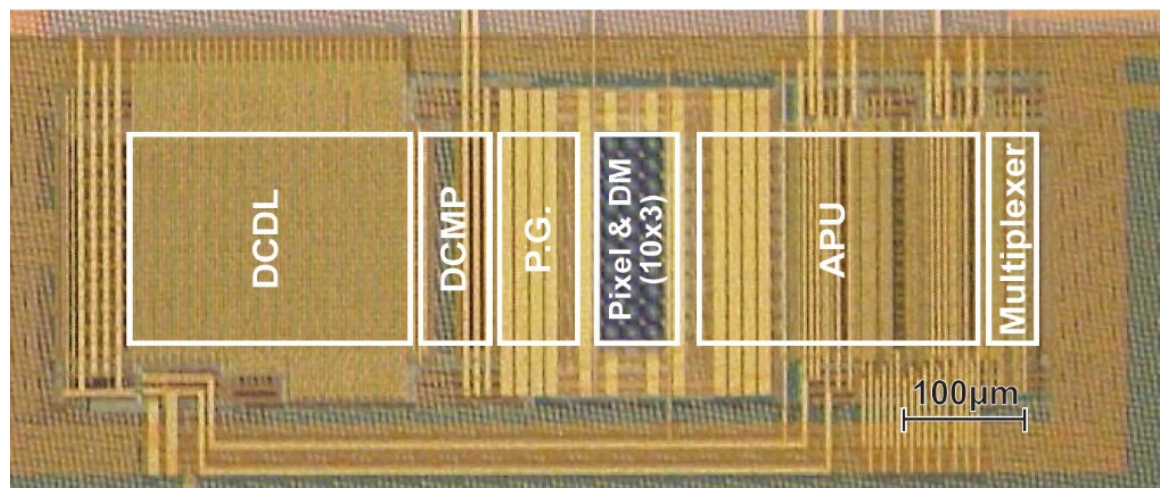

Figure 12. A photomicrograph of the prototype chip.

Figure 13 shows the measurement setup for demonstrating the proposed ToF measurement system using the time-domain feedback technique. A specific channel multiplexed from the APU outputs of the implemented chip is connected to an external comparator, and the bit-stream signal output from the comparator is connected to the DPU implemented in the FPGA. The output of the DPU as a 5-bit digital code is fed back to the DCDL of the chip and controls the gating-pulse delay of the demodulator (DM). The FPGA also supplies the light source triggering pulse and operation clock signals for the CSA, APU, and multiplexer of the chip. The DPU implemented in the FPGA covers the function of a counter, a time-offset adder, a decimation filter, and a JTAG interface to PC. The digital output of the chip is finally transferred to the PC for characterizing the implemented ToF sensor chip.

For demonstrating the ToF measurement using the proposed technique with timedomain feedback, a laser diode with a pulse width of $5 \mathrm{~ns}$ and a wavelength of $473 \mathrm{~nm}$ is used for the light source. The number of light pulses in each DSM oversampling period $N_{a}$ is 500 . The oversampling frequency used for the DSM is $4.2 \mathrm{MHz}$. The total ToF measurement time, including both coarse and fine measurements, is $22.4 \mathrm{~ms}$. The total DCDL delay is set to $7 \mathrm{~ns}$ with a unit step of $218 \mathrm{ps}$, and the maximum ToF measurement range is set to $105 \mathrm{~cm}$. A digital delay generator delays and sweeps the trigger pulse of the light source, $T_{L}$, to characterize the response of the implemented ToF measurement system to the pseudo-distance. The maximum number of DTC delay steps $N_{C}$ is set to 32 for a 5-bit coarse conversion. The total sampling number $N_{F}$ is set to 64 and 66 for the fine DSM conversion. Figure 14 shows a conceptual operating waveform of $Y_{\text {coarse }}, Y_{\text {fine }}$, and the combined output $Y_{\text {TOF }}$ for $N_{F}$ of 64 (Figure 14a) and 66 (Figure 14b). Although $N_{F}$ of 64 exactly corresponds to the 6-bit fine ToF-to-digital conversion, it causes time-variant errors at specific codes when the coarse and fine conversion codes are connected. This is because a discontinuous step appears in the linearity curve at the boundary of the maximum $\Delta T o F$. At this dis-continuous point, a time-variant error occurs, and this extraordinarily worsens the range resolution (or depth noise). This can be avoided by increasing $N_{F}$ to be larger than 64 . To address this issue, $N_{F}$ of 66 , corresponding to the $6^{+}$bits, is also evaluated for improving the depth resolution in Figure 14b. By increasing $N_{F}$ to be 66 , the gain of $Y_{\text {fine }}$ to ToF is increased by a factor of $66 / 64$ and the linearity curve of $Y_{\text {fine }}$ and the resulting 
$Y_{\text {ToF }}$ becomes as shown in Figure 14b. The discontinuous step in the linearity curve of $Y_{\text {ToF }}$ disappears, and the extraordinal depth noise at the discontinuous point is also reduced at the penalty of a little worse nonlinearity.

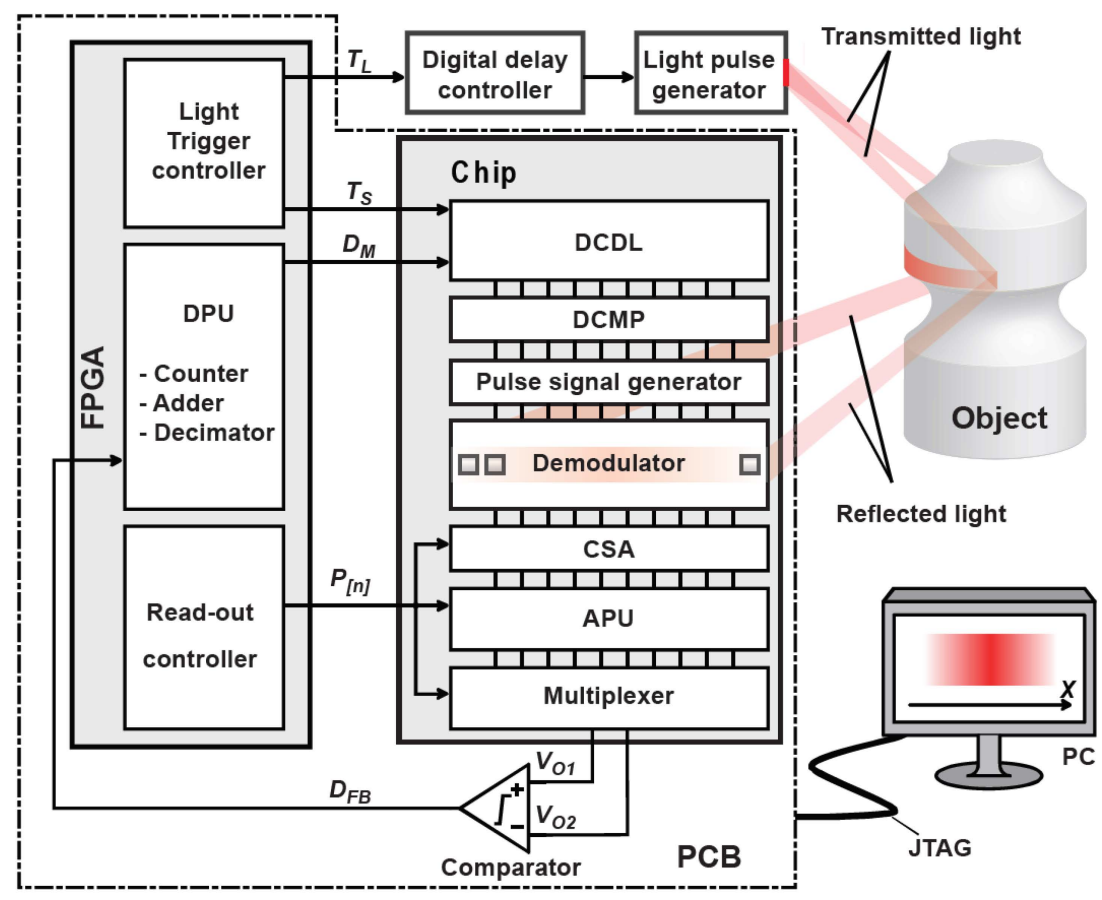

Figure 13. A measurement setup for demonstrating the proposed ToF measurement system.

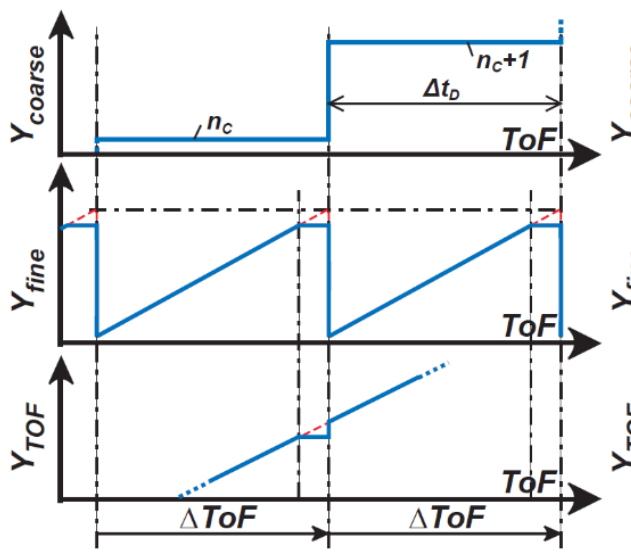

(a) $N_{F}=64$

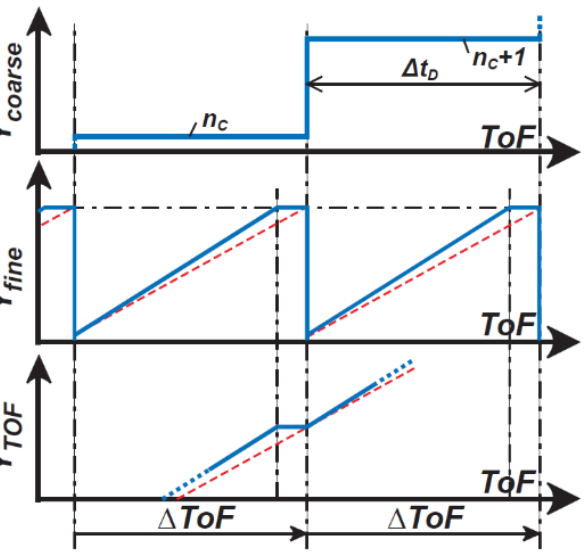

(b) $N_{F}=66$

Figure 14. A conceptual operating waveform of $Y_{\text {coarse }}, Y_{\text {fine, }}$, and the combined output $Y_{\text {ToF }}$ for $N_{F}$ of (a) 64 and (b) 66 .

Figures 15 and 16 show the results of the measured distance, differential nonlinearity (DNL), and integral nonlinearity (INL) for $N_{F}$ of 64 and $N_{F}$ of 66 , respectively. A good linearity is obtained without any corrections for linearity improvements. The maximum INL for the case of $N_{F}$ of 64 is $+0.9 \% /-0.47 \%(+0.94 \mathrm{~cm} /-0.49 \mathrm{~cm})$ to the entire range of $105 \mathrm{~cm}$. The maximum DNL for $N_{F}$ of 64 is $+0.43 \% /-0.76 \%(+0.45 \mathrm{~cm} /-0.8 \mathrm{~cm})$ to the entire range of $105 \mathrm{~cm}$. For the case of $N_{F}=66$, which is used for improving temporal errors, the maximum INL $(+1.29 \% /-0.51 \%(+1.36 \mathrm{~cm} /-0.54 \mathrm{~cm}))$ is a little worse than that for $N_{F}$ of 64 because the code resolution is not equal to 6 bits. However, the maximum DNL $(+0.33 \% /-0.58 \%(+0.35 \mathrm{~cm} /-0.6 \mathrm{~cm}))$ for the $N_{F}$ of 66 is even better than $N_{F}$ of 64 because the redundancy of the code ( $N_{F}$ of 66 corresponds to a resolution of 6.044 bits) might improve the nonlinearity at the connecting points of the coarse and fine digital codes. 


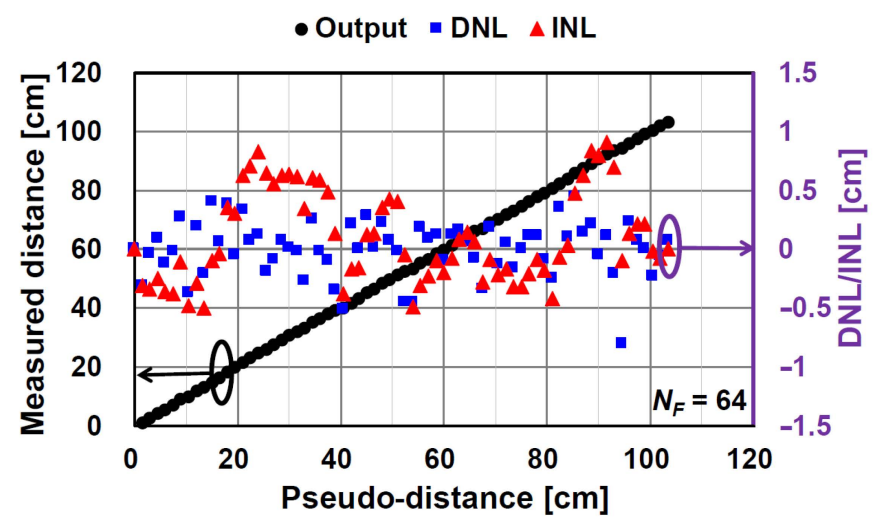

Figure 15. Measurement results of measured distance, differential nonlinearity (DNL), and integral nonlinearity (INL) of prototype chip $\left(N_{F}=64\right)$.

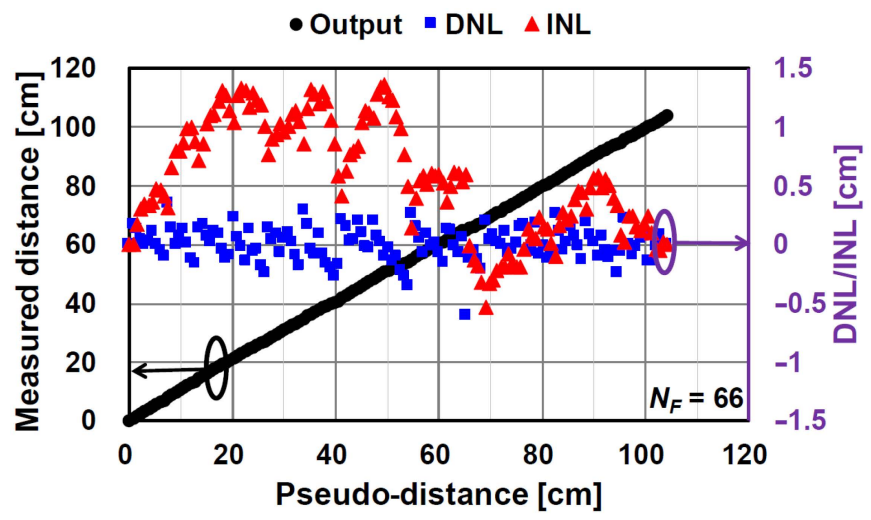

Figure 16. Measurement results of measured distance, DNL, and INL $\left(N_{F}=66\right)$.

Figure 17 shows the measurement results of the depth resolution to the pseudodistance. Two cases of fine ToF measurement setting for $N_{F}$ of 64 and $N_{F}$ of 66 were compared. For $N_{F}=64$, the range resolution of $0.27 \mathrm{~mm}$ (median) is good compared with other indirect ToF sensors in Table 1. However, at specific points, the range resolution is 3 times worse than its median, and the worst case is $1.02 \mathrm{~mm}$. This extra-ordinal error is because of the temporal variation of nonlinearity at the connection points of the coarse and fine digital code. This problem is solved by increasing the effective number of bits for fine measurement using DSM. By using $N_{F}$ of 66 , the range resolution (median) is improved from $0.27 \mathrm{~mm}$ to $0.24 \mathrm{~mm}$, and the worst value is from $1.02 \mathrm{~mm}$ to $0.37 \mathrm{~mm}$. Therefore, the code redundancy for fine ToF measurements might improve the temporal variation of nonlinearity at the connecting points of the coarse and fine digital codes, and the resulting depth resolution is entirely good.

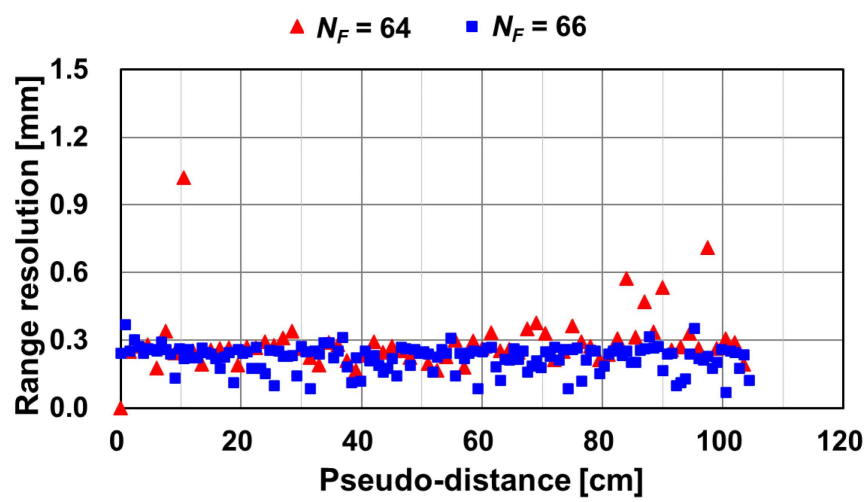

Figure 17. Measurement results of the depth resolution for $N_{F}=64$ and $N_{F}=66$. 
Table 1. Performance summary and comparison.

\begin{tabular}{|c|c|c|c|c|c|c|}
\hline & This Work & $\begin{array}{c}\left.\text { JSSC'19 }^{\prime} 126\right]\end{array}$ & $\begin{array}{c}\text { JSSC'19 } \\
\text { [15] }\end{array}$ & $\begin{array}{c}\text { JEDC'15 } \\
{[22]}\end{array}$ & $\begin{array}{c}\text { JSSC'15 } \\
\text { [10] }\end{array}$ & $\begin{array}{c}\text { Sensors'20 } \\
\text { [23] }\end{array}$ \\
\hline Array Type & Linear (1D) & Linear (1D) & Area (2D) & Area (2D) & Area (2D) & Area (2D) \\
\hline Process & $0.11 \mu \mathrm{m}$ FSI & $0.11 \mu \mathrm{m}$ FSI & 67 nm BSI & $0.11 \mu \mathrm{m}$ FSI & $0.13 \mu \mathrm{m}$ FSI & $0.11 \mu \mathrm{m}$ FSI \\
\hline ToF Type & Indirect SP & Indirect SP & Indirect CW & Indirect SP & Indirect CW & Indirect SP \\
\hline Readout & Time-Domain & Analog + & Analog + & Analog + & Analog + & Analog + \\
\hline Architecture & Feedback & Column ADC & Column ADC & Column ADC & Column ADC & Column ADC \\
\hline Pixel Pitch & $16.8 \mu \mathrm{m}$ & $22.4 \mu \mathrm{m}$ & $7 \mu \mathrm{m}$ & $16.8 \mu \mathrm{m}$ & $10 \mu \mathrm{m}$ & $22.4 \mu \mathrm{m}$ \\
\hline Pixel Number & $3(\mathrm{~V}) \times 10(\mathrm{H})$ & $8(\mathrm{~V}) \times 257(\mathrm{H})$ & $\begin{array}{c}480(\mathrm{~V}) \times \\
640(\mathrm{H})\end{array}$ & $\begin{array}{c}240(\mathrm{~V}) \times \\
413(\mathrm{H})\end{array}$ & $\begin{array}{c}424(\mathrm{~V}) \times \\
512(\mathrm{H})\end{array}$ & $\begin{array}{c}128(\mathrm{~V}) \times \\
134(\mathrm{H})\end{array}$ \\
\hline Pixel Type & 2-Tap & 3-Tap & 4-Tap & 3-Тар & 2-Тар & 8-Tap \\
\hline $\begin{array}{l}\text { Light } \\
\text { Wavelength } \\
\text { Modulation }\end{array}$ & $473 \mathrm{~nm}$ & $473 \mathrm{~nm}$ & $940 \mathrm{~nm}$ & $870 \mathrm{~nm}$ & $860 \mathrm{~nm}$ & $850 \mathrm{~nm}$ \\
\hline $\begin{array}{c}\text { Frequency } \\
\text { (Pulse Width) }\end{array}$ & $5 \mathrm{~ns}$ & $<80$ ps & $100 \mathrm{MHz}$ & $13 \mathrm{~ns}$ & $10-130 \mathrm{MHz}$ & $6 \mathrm{~ns}$ \\
\hline Range & $\begin{array}{l}\text { (pseudo) } \\
0-1.05 \mathrm{~m}\end{array}$ & $0-25 \mathrm{~mm}$ & $0.4-4 \mathrm{~m}$ & $0.8-1.8 \mathrm{~m}$ & $0.8-4.2 \mathrm{~m}$ & $1-6.4 \mathrm{~m}$ \\
\hline Nonlinearity & $\begin{array}{c}+0.9 \% /-0.5 \% \\
\quad(0.94 \mathrm{~cm})\end{array}$ & $\begin{array}{c}<1 \% \\
(<0.25 \mathrm{~mm})\end{array}$ & - & $\begin{array}{c}1.9 \% \\
(<2 \mathrm{~cm})\end{array}$ & - & $\begin{array}{c}0.67 \% \\
(<4 \mathrm{~cm})\end{array}$ \\
\hline $\begin{array}{c}\text { Resolution } \\
\quad(\mathrm{rms}) \\
\text { (\% to full range) }\end{array}$ & $\begin{array}{l}0.27 \mathrm{~mm} \\
(0.026 \%)\end{array}$ & $\begin{array}{c}64 \mu \mathrm{m} \\
(0.256 \%)\end{array}$ & $<0.62 \%$ & $\begin{array}{l}7 \mathrm{~mm} \\
(0.7 \%)\end{array}$ & $\begin{array}{c}15 \mathrm{~mm} @ 3.5 \mathrm{~m} \\
(0.441 \%)\end{array}$ & $\begin{array}{c}8 \mathrm{~mm} \\
(0.148 \%)\end{array}$ \\
\hline
\end{tabular}

The performance summary of the ToF sensor of this work and a comparison to other works are shown in Table 1 . Because the implemented ToF sensor chip is a preliminary one having only $3(\mathrm{~V}) \times 10(\mathrm{H})$ pixels and the design specifications are different from each other, this table is not intended for the overall chip performance comparison. With this table, however, the important feature of this work is clarified by the comparison of linearity and range resolution. In general, meeting both high linearity and high range resolution is difficult. A high range resolution needs a high-speed response of the pixel to use shorter light pulse modulation or higher modulation frequency, leading to distortions in the light-pulse waveform, photo-current waveform, and analog demodulation. The proposed technique using the time-domain negative feedback in this work is the best method for simultaneously meeting high linearity $(+0.9 \% /-0.5 \%)$ and high range resolution $(0.27 \mathrm{~mm}$ (median), $0.026 \%$ to full range $(1.05 \mathrm{~m})$ ). Particularly for the comparison with Reference [26], which demonstrated sub-100 $\mu \mathrm{m}$ resolution, the advantage of this work is the large working range while attaining a good resolution. This feature comes from the proposed coarse-to-fine ToF measurement method, where the range is digitally extended by the incremental DTC and resolved finely with the short pulse width of $5 \mathrm{~ns}$ and oversampled noise suppression in the delta-sigma modulation.

Table 2 shows comparison of ToF sensor architectures including this work and two other typical ToF sensor architectures. The indirect ToF sensors using CW (Continuous Wave) and SP (Short Pulse) modulation uses simple analog readout circuits like a source follower. Because of this, it is suitable for implementing large area-array (2D pixel array)type ToF image sensors. However, the analog readout types have issues of the nonlinearity and stability due to the analog elements like a photo-demodulator and amplifier. Because the role of the indirect ToF pixel is just the demodulation of modulated photo signals, the processing for range calculation and ambient light canceling have to be done by postprocessing at an external system. In contrast to the indirect ToF sensors using analog readout circuits, the readout circuits per pixel of the direct ToF sensor using the SPAD and this work are based on digital-processing techniques. Though the readout and processing circuits are relatively complicated when compared with those of the indirect ToF sensors, there is a distinct advantage in its principle for having a high accuracy (high linearity) because of the use of the digitally regulated time reference. The direct ToF sensor using time 
stamping and the digital ToF sensor using the time-domain feedback technique (this work) use a time-to-digital converter and digital-to-time converter, respectively, for measuring the $\mathrm{ToF}$, and the accuracy (linearity) is dominated by these digital elements, whose accuracy and stability can be much better than the analog counterparts. In these direct ToF sensors, the range calculation and the ambient-light cancellation are self-contained because the digital output is just the ToF itself. The biggest advantage of the direct ToF sensor using SPAD lies in its single-photon sensitivity, and therefore the SPAD-based direct ToF sensors are becoming a key technology for long-distance $(>50 \mathrm{~m})$ LiDAR applications. On the other hand, its photon-based processing may cause a difficulty in treating many photons, and the dynamic range is limited by the processing speed of the time-stamping of a photon. Therefore, the SPAD-based direct ToF sensors are not always the best solution for distance measurements for a few meters and with strong signals. The per-pixel readout circuits of the digital ToF sensor using the time-domain feedback are more complicated than that of the indirect ToF sensors but less complicated than that of the SPAD-based direct ToF sensors. The ambient light canceling is done by an analog fully differential charge-sensitive amplifier (CSA) during the demodulation process. While the dynamic range is limited by the full well capacity (FWC) of the fully differential CSA, the use of oversampling done in the delta-sigma modulation is effective for extending the dynamic range and reducing the influence of shot noise, because the integration of the sampled CSA outputs in the integrator has an effect of improving the shot-noise-limited signal-to-noise ratio. As a result, high range resolution $(0.27 \mathrm{~mm} @ 0-1.05 \mathrm{~m})$ is attained in this work. Because of the complicated analog and digital circuits per pixel, the proposed circuits architecture is suitable only for linear-array (1D) ToF image sensors.

Table 2. Comparison of ToF Sensor Architectures.

\begin{tabular}{|c|c|c|c|}
\hline & $\begin{array}{c}\text { Indirect ToF Using CW or SP } \\
\text { Modulation }\end{array}$ & Direct ToF Using SPAD & $\begin{array}{l}\text { This Work (Digital ToF with } \\
\text { Time-Domain Feedback) }\end{array}$ \\
\hline Readout circuits per pixel & $\begin{array}{l}\text { - Analog } \\
\text { - Simple }\end{array}$ & $\begin{array}{l}\text { - Digital (Time Stamping) } \\
\text { - Complicated }\end{array}$ & $\begin{array}{l}\text { - Digital (Time-Domain } \\
\text { Feedback) } \\
\text { - Complicated }\end{array}$ \\
\hline What limits the linearity & $\begin{array}{l}\text { - Analog Elements } \\
\text { (Demodulator \& Amplifier) }\end{array}$ & $\begin{array}{l}\text { - Digital Elements } \\
\text { (Time-to-Digital Converter) }\end{array}$ & $\begin{array}{l}\text { - Digital Elements } \\
\text { (Digital-to-Time Converter) }\end{array}$ \\
\hline Ambient light canceling & - Post Processing & $\begin{array}{l}\text { - On chip } \\
\text { - Sophisticated but } \\
\text { Complicated } \\
\text { - Digital Processing }\end{array}$ & $\begin{array}{l}\text { - On chip } \\
\text { - Simple } \\
\text { - Analog Processing }\end{array}$ \\
\hline Range calculation & - Post Processing & - Self-Contained & - Self-Contained \\
\hline $\begin{array}{l}\text { What determines the dynamic } \\
\text { range }\end{array}$ & - FWC in FDA & $\begin{array}{l}\text { - Photon-Based Processing } \\
\text { - Pile-up of Photon Signals }\end{array}$ & $\begin{array}{l}\text { - FWC in CSA } \\
\text { - Oversampling Ratio }\end{array}$ \\
\hline Suitability to pixel-array type & $\begin{array}{l}\text { - Linear Pixel-Array (1D) } \\
\text { - Area Pixel-Array (2D) }\end{array}$ & $\begin{array}{l}\text { - Linear Pixel-Array (1D) } \\
\text { - Area Pixel-Array (2D) }\end{array}$ & - Linear Pixel-Array (1D) \\
\hline $\begin{array}{l}\text { Suitability to weak-signal } \\
\text { application }\end{array}$ & - Fair & - Good & - Fair \\
\hline Range resolution (Precision) & $0.148 \%$ (8 mm) @ (1-6.4m) [23] & $1.4 \mathrm{~mm} @(2 \mathrm{~m}-50 \mathrm{~m})[5]$ & $\begin{array}{l}0.27 \mathrm{~mm} @(0-1.05 \mathrm{~m}, \text { Pseudo } \\
\text { Distance) }\end{array}$ \\
\hline
\end{tabular}

\section{Conclusions}

A high-linearity high-depth resolution ToF linear-array digital image sensor using time-domain feedback that works as incremental gating-pulse delay feedback and DSM for coarse and fine ToF measurements, respectively, has been presented. The coarse ToF measurement uses time-domain feedback using the gating-pulse delay generated by a 5-bit DTC. The fine ToF measurement uses a first-order DSM for time-domain bit-stream 
feedback and oversampling signal processing for attaining high piecewise linearity and low depth noise (high depth resolution).

For a demonstration of the proposed system, a prototype sensor chip was implemented with a $0.11 \mu \mathrm{m}(1 \mathrm{P} 4 \mathrm{M})$ CIS process. The chip contains a DTC, pulse generator, demodulation pixels, APU, and multiplexer. The other necessary functional blocks, such as a comparator, the DPU, timing controller, light source, and digital delay generator, are implemented using discrete components or an FPGA. The ToF measurement system produces an 11-bit fully digital output with a measurement cycle time of $22 \mathrm{~ms}$. The prototype ToF system observed good linearity of $+0.9 \% /-0.47 \%$. An excellent range resolution of $0.24 \mathrm{~mm}$ (best case) for the range of $0-1.05 \mathrm{~m}$ is demonstrated. The residual nonlinearity is predominantly because of the deviation of the delay elements in the implemented DTC, which will be improved by introducing a precise DTC.

Author Contributions: J.K. and S.K. proposed the device concept and K.K. and S.K. provided the overall guidance of the project. J.K. designed and measured the chip. J.K., K.Y., and S.K. drafted the manuscript. All authors have read and agreed to the published version of the manuscript.

Funding: This work was supported in part by the Japan Society for the Promotion of Science (JSPS) KAKENHI, the Grant-in-Aid for Scientific Research (S) under Grant Number 18H05240 through the Ministry of Education, Culture, Sports, Science, and Technology (MEXT), the JST COI Program under Grant Number JPMJCE1311(MEXT), and the Program for Building Regional Innovation Ecosystems (MEXT).

Institutional Review Board Statement: Not applicable.

Informed Consent Statement: Not applicable.

Data Availability Statement: Data available in a publicly accessible repository.

Acknowledgments: VLSI Design and Education Center (VDEC), The University of Tokyo with the collaboration with Cadence Corporation, and the authors appreciate Dongbu HiTek for the CIS fabrication.

Conflicts of Interest: The authors declare no conflict of interest.

\section{References}

1. Shcherbakova, O.; Pancheri, L.; Dalla Betta, G.F.; Massari, N.; Stoppa, D. 3D camera based on linear-mode gain-modulated avalanche photodiodes. In Proceedings of the 2013 IEEE International Solid-State Circuits Conference Digest of Technical Papers (ISSCC 2013), San Francisco, CA, USA, 17-21 February 2013; Volume 56, pp. 490-491. [CrossRef]

2. Akita, H.; Takai, I.; Azuma, K.; Hata, T.; Ozaki, N. An imager using 2-D single-photon avalanche diode array in 0.18- $\mu \mathrm{m}$ CMOS for automotive LIDAR application. IEEE Symp. VLSI Circuits Dig. Tech. Pap. 2017, 2, C290-C291. [CrossRef]

3. Lange, R.; Seitz, P. Solid-state time-of-flight range camera. IEEE J. Quantum Electron. 2001, 37. [CrossRef]

4. Niclass, C.; Soga, M.; Matsubara, H.; Ogawa, M.; Kagami, M. A 0.18-m cmos soc for a 100-m-range 10-frame/s $200 \times 96-$ pixel time-of-flight depth sensor. IEEE J. Solid State Circuits 2014, 49, 315-330. [CrossRef]

5. Zhang, C.; Lindner, S.; Antolovic, I.M.; Mata Pavia, J.; Wolf, M.; Charbon, E. A 30-frames/s, $252 \times 144$ SPAD Flash LiDAR with 1728 Dual-Clock 48.8-ps TDCs, and Pixel-Wise Integrated Histogramming. IEEE J. Solid State Circuits 2019, 54, $1137-1151$. [CrossRef]

6. Ximenes, A.R.; Padmanabhan, P.; Lee, M.J.; Yamashita, Y.; Yaung, D.N.; Charbon, E. A $256 \times 256$ 45/65 nm 3D-stacked SPADbased direct TOF image sensor for LiDAR applications with optical polar modulation for up to $18.6 \mathrm{~dB}$ interference suppression. In Proceedings of the Digest of Technical Papers-IEEE International Solid-State Circuits Conference, San Francisco, CA, USA, 11-15 February 2018; Volume 61.

7. Kondo, S.; Kubota, H.; Katagiri, H.; Ota, Y.; Hirono, M.; Ta, T.T.; Okuni, H.; Ohtsuka, S.; Ojima, Y.; Sugimoto, T.; et al. A $240 \times 192$ Pixel 10 fps 70 klux 225 m-Range Automotive LiDAR SoC Using a 40ch 0.0036 mm 2 Voltage/Time Dual-Data-Converter-Based AFE. In Proceedings of the 2020 IEEE International Solid- State Circuits Conference-(ISSCC), San Francisco, CA, USA, 16-20 February 2020; pp. 94-96.

8. Spirig, T.; Seitz, P.; Vietze, O.; Heitger, F. The Lock-In CCD—Two-Dimensional Synchronous Detection of Light. IEEE J. Quantum Electron. 1995, 31, 1705-1708. [CrossRef]

9. Spirig, T.; Seitz, P. The multitap lock-in ccd with offset subtraction. IEEE Trans. Electron Devices 1997, 44. [CrossRef]

10. Bamji, C.S.; O'Connor, P.; Elkhatib, T.; Mehta, S.; Thompson, B.; Prather, L.A.; Snow, D.; Akkaya, O.C.; Daniel, A.; Payne, A.D.; et al. A $0.13 \mu \mathrm{m}$ CMOS System-on-Chip for a $512 \times 424$ Time-of-Flight Image Sensor with Multi-Frequency Photo-Demodulation up to $130 \mathrm{MHz}$ and $2 \mathrm{GS} / \mathrm{s}$ ADC. IEEE J. Solid State Circuits 2015, 50, 303-319. [CrossRef] 
11. Stoppa, D.; Massari, N.; Pancheri, L.; Malfatti, M.; Perenzoni, M.; Gonzo, L. A range image sensor based on 10- $\mu \mathrm{m}$ lock-in pixels in $0.18 \mu \mathrm{m}$ CMOS imaging technology. IEEE J. Solid State Circuits 2011, 46, 248-258. [CrossRef]

12. Kim, S.J.; Kim, J.D.K.; Kang, B.; Lee, K. A CMOS image sensor based on unified pixel architecture with time-division multiplexing scheme for color and depth image acquisition. IEEE J. Solid State Circuits 2012, 47, 2834-2845. [CrossRef]

13. Bamji, C.S.; Mehta, S.; Thompson, B.; Elkhatib, T.; Wurster, S.; Akkaya, O.; Payne, A.; Godbaz, J.; Fenton, M.; Rajasekaran, V.; et al. IMpixel 65nm BSI 320MHz demodulated TOF Image sensor with $3 \mu \mathrm{m}$ global shutter pixels and analog binning. In Proceedings of the Digest of Technical Papers-IEEE International Solid-State Circuits Conference, San Francisco, CA, USA, 11-15 February 2018; Volume 61.

14. Kato, Y.; Sano, T.; Moriyama, Y.; Maeda, S.; Yamazaki, T.; Nose, A.; Shiina, K.; Yasu, Y.; Van Der Tempel, W.; Ercan, A.; et al. $320 \times$ 240 Back-Illuminated 10- $\mu$ m CAPD Pixels for High-Speed Modulation Time-of-Flight CMOS Image Sensor. IEEE J. Solid State Circuits 2018, 53. [CrossRef]

15. Keel, M.S.; Jin, Y.G.; Kim, Y.; Kim, D.; Kim, Y.; Bae, M.; Chung, B.; Son, S.; Kim, H.; An, T.; et al. A VGA Indirect Time-of-Flight CMOS Image Sensor with 4-Tap 7- $\mu \mathrm{m}$ Global-Shutter Pixel and Fixed-Pattern Phase Noise Self-Compensation. IEEE J. Solid State Circuits 2020, 55, 889-897. [CrossRef]

16. Kawahito, S.; Halin, I.A.; Ushinaga, T.; Sawada, T.; Homma, M.; Maeda, Y. A CMOS time-of-flight range image sensor with gates-on-field-oxide structure. IEEE Sens. J. 2007, 7. [CrossRef]

17. Sawada, T.; Kawahito, S.; Nakayama, M.; Ito, K.; Halin, I.A.; Homma, M.; Ushinaga, T.; Maeda, Y. A TOF range image sensor with an ambient light charge drain and small duty-cycle light pulse. In Proceedings of the 2007 International Image Sensor Workshop, Ogunquit, ME, USA, 7-10 June 2007; pp. 254-257.

18. Spickermann, A.; Durini, D.; Süss, A.; Ulfig, W.; Brockherde, W.; Hosticka, B.J.; Schwope, S.; Grabmaier, A. CMOS 3D image sensor based on pulse modulated time-of-flight principle and intrinsic lateral drift-field photodiode pixels. Eur. Solid State Circuits Conf. 2011, 111-114. [CrossRef]

19. Lee, S.; Yasutomi, K.; Morita, M.; Kawanishi, H.; Kawahito, S. A time-of-flight range sensor using four-tap lock-in pixels with high near infrared sensitivity for lidar applications. Sensors 2020, 20, 116. [CrossRef]

20. Kondo, K.; Yasutomi, K.; Yamada, K.; Komazawa, A.; Handa, Y.; Okura, Y.; Michiba, T.; Aoyama, S.; Kawahito, S. A Built-in Drift-field PD Based 4-tap Lock-in Pixel for Time-of-Flight CMOS Range Image Sensors. In Proceedings of the Extended Abstracts of the 2018 International Conference on Solid State Devices and Materials, Tokyo, Japan, 9-13 September 2018; pp. 9-13.

21. Trang, H.; Kasugai, T.; Isobe, K.; Han, S.M.; Takasawa, T.; Lioe, D.X.; Yasutomi, K.; Kagawa, K.; Kawahito, S. Design, implementation and evaluation of a TOF range image sensor using multi-tap lock-in pixels with cascaded charge draining and modulating gates. Electron. Imaging 2016, 2016, 1-6. [CrossRef]

22. Han, S.M.; Takasawa, T.; Yasutomi, K.; Aoyama, S.; Kagawa, K.; Kawahito, S. A time-of-flight range image sensor with background canceling lock-in pixels based on lateral electric field charge modulation. IEEE J. Electron Devices Soc. 2015, 3, 267-275. [CrossRef]

23. Shirakawa, Y.; Yasutomi, K.; Kagawa, K.; Aoyama, S.; Kawahito, S. An 8-tap CMOS lock-in pixel image sensor for short-pulse time-of-flight measurements. Sensors 2020, 20, 1040. [CrossRef]

24. Kim, D.; Lee, S.; Park, D.; Piao, C.; Park, J.; Ahn, Y.; Cho, K.; Shin, J.; Song, S.M.; Kim, S.J.; et al. A Dynamic Pseudo 4-Tap CMOS Time-of-Flight Image Sensor with Motion Artifact Suppression and Background Light Cancelling over 120 klux. In Proceedings of the Digest of Technical Papers-IEEE International Solid-State Circuits Conference, San Francisco, CA, USA, 16-20 February 2020.

25. Yasutomi, K.; Usui, T.; Han, S.M.; Takasawa, T.; Kagawa, K.; Kawahito, S. A submillimeter range resolution time-of-flight range imager with column-wise skew calibration. IEEE Trans. Electron Devices 2016, 63, 182-188. [CrossRef]

26. Yasutomi, K.; Okura, Y.; Kagawa, K.; Kawahito, S. A Sub-100 $\mu \mathrm{m}$-Range-Resolution Time-of-Flight Range Image Sensor With Three-Tap Lock-In Pixels, Non-Overlapping Gate Clock, and Reference Plane Sampling. IEEE J. Solid State Circuits 2019, 54, 2291-2303. [CrossRef]

27. Cruz, J.B.; Perkins, W.R. A New Approach to the Sensitivity Problem in Multivariable Feedback System Design. IEEE Trans. Automat. Contr. 1964, 9. [CrossRef]

28. Tang, Y.; Geiger, R.L. Effects of open-loop nonlinearity on linearity of feedback amplifiers. In Proceedings of the 2002 IEEE International Symposium on Circuits and Systems, Scottsdale, AZ, USA, 26-29 May 2002; Volume 2, pp. 2-5. [CrossRef]

29. Agrawal, B.; Shenoi, K. Design methodology for $\Sigma \Delta$ M. IEEE Trans. Commun. 1983, 31, 360-370. [CrossRef]

30. Schreier, R.; Temes, G.C. Understanding Delta-Sigma Data Converters; IEEE Press: Piscataway, NJ, USA, 2005 ; Volume 74.

31. Eynde, F.O.; Sansen, W.M.C. Analog Interfaces for Digital Signal Processing Systems; Springer Science \& Business Media: Berlin/Heidelberg, Germany, 2012; Volume 225, ISBN 1461532566. 